\title{
Efeito da Estação de Monta e do Tipo de Cruzamento sobre o Desempenho de Cabras na Região Semi-Árida do Nordeste do Brasil ${ }^{1}$
}

João Bandeira de Moura Neto $^{2}$, José Nilton Moreira ${ }^{3}$, Daniel Maia Nogueira ${ }^{3}$, Tadeu Vinhas Voltolini ${ }^{3}$, Chirles Araújo de França ${ }^{2}$

Resumo: O objetivo desse trabalho foi avaliar o efeito da estação de monta e do tipo de cruzamento sobre o desempenho produtivo e reprodutivo de cabras criadas em sistema de produção agroecológico, na região semiárida do Nordeste do Brasil. Foram utilizadas 47 matrizes, em média, avaliadas em três estações de monta (EM) durante dois anos, como a seguir: EM1 (março/abril de 2005), EM2 (novembro/dezembro de 2005) e EM3 (julho/agosto de 2006). Foram utilizados os seguintes cruzamentos (reprodutor x matrizes): Boer x sem raça definida (SRD), Anglo-nubiano x SRD, Canindé x Canindé ou Repartida x Repartida. Não foi observada diferença significativa $(\mathrm{P}>0,05)$ para o período da estação de monta nem para o tipo de cruzamento sobre a fertilidade ao parto, prolificidade e taxa de reprodução. No entanto, houve efeito $(\mathrm{P}<0,05)$ da estação de monta e do tipo de cruzamento sobre o peso das crias ao nascimento e aos 90 dias de idade. As crias provenientes do cruzamento da raça Boer x SRD ou Anglo x SRD apresentaram maior $(\mathrm{P}<0,05)$ peso ao nascimento e aos 90 dias que as crias das raças nativas. Foi observado um intervalo entre partos médio de 10 meses (1,2 partos/ ano). A utilização de cruzamentos industriais pode ser uma alternativa para melhorar o desempenho produtivo de caprinos explorados na região semi-árida do Nordeste do Brasil.

Palavras-chave: caprinos, estação de monta, fertilidade, prolificidade, reprodução

\section{Effect of Mating Season and Type of Breeding Over the Productive Performance of Goats Raised in the Semi-Arid Zone of Northeastern Brazil}

\begin{abstract}
This work aimed to evaluate the effect of mating season and type of breed on the reproductive performance of goats raised in an agroecological production system in the semi-arid region of Northeastern Brazil. An average of 47 goats were evaluated for three mating season (EM) during two years, as following: EM1 (Mach/April, 2005), EM2 (November/December, 2005) and EM3 (July/August, 2006). The types of breeding (bulk x females) used were: Boer x undefined genotype (SRD), Anglo- nubiano x SRD, Caninde x Caninde or Repartida $x$ Repartida. Significant difference was not observed $(\mathrm{P}>0.05)$ in the mating season nor for type of breeding on fertility rate, prolificity and reproduction rate. However, there was effect $(\mathrm{P}<0.05)$ for the mating season and for type of breeding on kids' life weight at birth and at 90 days old. The kids coming from Boer $\mathrm{x}$ SRD and Anglo x SRD showed larger $(\mathrm{P}<0.05)$ life weigh at birth and at 90 days old. It was found a kidding interval of 10 months (1.2 kids/ parturition). The use of exotic breed can be an alternative to improve the productive performance of goats explored in the semi-arid area of the Northeast of Brazil.
\end{abstract}

Key words: fertility, goats, mating season, prolificity, reproduction

\footnotetext{
${ }^{1}$ Pesquisa financiada pelo Ministério da Integração Nacional

${ }^{2}$ Pesquisador da Embrapa Semi-Árido - BR 428, Km 152. Zona Rural, CP 23, CEP. 56300-970, BR 428, Km 152, Zona Rural. Petrolina, PE, Brasil. E-mails para correspondência: daniel@cpatsa.embrapa.br ou tadeu.voltolini@cpatsa.embrapa.br

${ }^{3}$ Mestrando Programa de Pós-Graduação em Ciência Animal - UNIVASF - Rodovia BR 407, Km 12 - Lote 543 - Projeto de Irrigação Senador Nilo Coelho, s/nº - C1 - CEP 56300-990 - Petrolina - PE, Brasil, Bolsista FACEPE
} 
Rev. Cient. Prod. Anim., v.12, n.1, p.60-63, 2010

\section{Introdução}

A caprinocultura na região Nordeste do Brasil é uma das principais atividades econômicas e importante fonte de proteína animal. Nesta região, os partos podem ocorrer durante todo o ano e essa dispersão dos nascimentos dificulta o manejo das crias, aumenta as taxas de mortalidade e forma lotes desuniformes para a comercialização. Além disso, o desempenho reprodutivo dos caprinos explorados na região semi-árida é caracterizado por baixos índices de fertilidade, prolificidade e alta taxa de mortalidade até o desmame (Guimarães Filho, 1983; Simplício et al., 1990).

Neste sentido, alguns esforços têm sido realizados visando melhorar os índices zootécnicos, sem alterar de forma significativa os sistemas de produção locais. Na Embrapa Semi-Árido trabalhou-se com um sistema de criação denominado "Cabrito Ecológico", em que os animais foram submetidos a estações de monta, em regime semi-intensivo de produção, com pouco uso de insumos externos e associados à vegetação da Caatinga (Holanda Júnior et al., 2004; Nogueira \& Holanda Júnior, 2005). A estação de monta permite uma maior uniformidade da produção, possibilitando a seleção, melhoramento e avaliação reprodutiva do rebanho. Além disso, uma das alternativas para incrementar a produção de carne é fazer o uso da diversidade genética do rebanho, através de cruzamentos entre diferentes raças. $\mathrm{O}$ objetivo desse trabalho foi avaliar o efeito da estação de monta e do tipo de cruzamento sobre o desempenho de cabras exploradas em um sistema de produção agroecológico, na região semiárida do Nordeste do Brasil.

\section{Material e Métodos}

$\mathrm{O}$ experimento foi conduzido durante o período de março de 2005 a fevereiro de 2007, no campo experimental da caatinga da Embrapa Semi-Árido, em Petrolina-PE.

Foram utilizadas, em média, 47 matrizes submetidas a três estações de monta em períodos préestabelecidos para a ocorrência de três partos em dois anos, como a seguir: EM1 (março/abril de 2005), EM2 (novembro/dezembro de 2005) e EM3 (julho/agosto de 2006). Foram utilizados os seguintes cruzamentos (reprodutor x matrizes): Boer x sem raça definida (SRD), Anglo-nubiano x SRD, Canindé x Canindé ou Repartida x Repartida. Durante as estações de monta, cada grupo de acasalamento permaneceu junto com o respectivo reprodutor durante a noite, sendo separado na manhã do dia seguinte.

Durante os períodos chuvosos, os animais tiveram acesso exclusivo à vegetação da caatinga, enquanto que nos períodos secos, os animais tiveram acesso às pastagens de capim Buffel (Cenchrus ciliares L.), recebendo feno de leucena (Leucaena leucocephala), silagem de maniçoba (Manihot glaziowii) e suplemento mineral, ao final da tarde.

Os parâmetros avaliados foram: fertilidade ao parto (cabras paridas/total de matrizes expostas na EM x 100), prolificidade (crias nascidas/cabras paridas), peso das crias ao nascimento e aos 90 dias de idade (desmame) e taxa de reprodução (crias desmamadas/ matrizes expostas na EM x 100).

Para análise estatística, os cruzamentos entre Canindé x Canindé e de Repartida x Repartida foram agrupados no acasalamento de cabras Nativas. Foi utilizado um delineamento inteiramente casualizado em arranjo fatorial $3 \times 3$ (três estações de monta $x$ três tipos de cruzamento). Os parâmetros de fertilidade ao parto e taxa de reprodução foram analisados pelo teste Qui-quadrado, enquanto os dados de prolificidade, peso das crias ao nascimento e aos 90 dias foram analisados pelo procedimento ANOVA, sendo as médias comparadas pelo teste de Tukey, adotando 5\% de probabilidade. 


\section{Resultados e Discussão}

Não houve diferença significativa $(\mathrm{P}>0,05)$ entre as estações de monta nem entre os tipos de cruzamento sobre a fertilidade ao parto, prolificidade e taxa de reprodução (Tabela 1).

A eficiência reprodutiva do rebanho é o parâmetro que, isoladamente, mais contribui para o aumento da produtividade, sendo necessário que as condições de ambiente para exploração, os custos de produção e mercado sejam favoráveis (Simplício, 1990).

Na Tabela 1 pode ser observada uma redução numérica da taxa de reprodução da EM1 e uma diminuição da fertilidade ao parto da EM2. Na EM1, a taxa de reprodução foi reduzida pelo aumento da taxa de mortalidade dos cabritos até o desmame, que chegou a $25 \%$ neste período. Na EM2, a fertilidade ao parto foi reduzida, provavelmente, pela não manifestação do estro das fêmeas ou por problemas na concepção. Este fato pode estar relacionado com a oferta quanti-qualitativa de forragem nesses períodos.

Portanto, se as cobrições (montas) ou os nascimentos das crias ocorrerem durante o período seco, poderá promover redução da fertilidade ou aumento da mortalidade, se não houver melhor suplementação nutricional.

Quanto à prolificidade, foi observada uma média de 1,3 cabritos/parto, estando dentro na média para sistemas explorados no Nordeste do Brasil. Holanda Júnior et al. (2004) trabalharam em condições semelhantes e encontraram valores mais elevados, chegando a 1,71 crias por parto.

Foi observado efeito $(\mathrm{P}<0,05)$ da estação de monta e do tipo de cruzamento sobre o peso das crias, bem como, o efeito da interação EM x tipo de cruzamento. As crias provenientes da EM2 apresentaram menores $(\mathrm{P}<0,05)$ pesos ao nascimento e aos 90 dias de idade quando comparada a EM3 (Tabela 1).

Tabela 1 - Número de matrizes expostas, fertilidade ao parto, prolificidade, peso ao nascimento e ao desmame (90 dias) e taxa de reprodução em função da estação de monta (EM) e do tipo de cruzamento

\begin{tabular}{|c|c|c|c|c|c|}
\hline \multirow{2}{*}{$\begin{array}{c}\text { Estação de } \\
\text { monta e partos }\end{array}$} & \multirow{2}{*}{ Coeficientes técnicos } & \multicolumn{3}{|c|}{ Tipo de cruzamento } & \multirow{2}{*}{$\begin{array}{c}\text { Interação } \\
\text { EM x Cruz }\end{array}$} \\
\hline & & Boer x SRD & Anglo x SRD & Nativas & \\
\hline \multirow{6}{*}{$\begin{array}{c}\text { EM1 } \\
\text { (março/abril) } \\
\text { Parto: } \\
\text { agosto/setembro }\end{array}$} & Matriz exposta, $n$ & 16 & 14 & 20 & \multirow{6}{*}{$\begin{array}{l}\mathrm{P}<0,05 \\
\mathrm{P}<0,05\end{array}$} \\
\hline & Fertilidade ao parto, $\%$ & 62,5 & 57,1 & 80,0 & \\
\hline & Prolificidadem $\mathrm{n}$ & $1,3^{\mathrm{a}}$ & $1,1^{\mathrm{a}}$ & $1,2^{\mathrm{a}}$ & \\
\hline & Peso ao nascimento, $\mathrm{kg}$ & $3,1^{\mathrm{a}}$ & $2,5^{\mathrm{b}}$ & $1,9^{\mathrm{c}}$ & \\
\hline & Peso aos 90 dias, $\mathrm{kg}$ & $13,5^{\mathrm{a}}$ & $10,8^{\mathrm{a}}$ & $6,9^{\mathrm{b}}$ & \\
\hline & Taxa de reprodução, \% & 42,8 & 50,0 & 65,0 & \\
\hline \multirow{6}{*}{$\begin{array}{c}\text { EM1 } \\
\text { (novembro/dezembro) } \\
\text { Parto: } \\
\text { abril } / \text { maio }\end{array}$} & Matriz exposta, $n$ & 14 & 14 & 20 & \multirow{6}{*}{$\begin{array}{l}\mathrm{P}<0,05 \\
\mathrm{P}<0,05\end{array}$} \\
\hline & Fertilidade ao parto, $\%$ & 50,0 & 57,5 & 40,0 & \\
\hline & Prolificidadem $n$ & $1,3^{\mathrm{a}}$ & $1,5^{\mathrm{a}}$ & $1,5^{\mathrm{a}}$ & \\
\hline & Peso ao nascimento, $\mathrm{kg}$ & $3,0^{\mathrm{a}}$ & $2,9^{\mathrm{a}}$ & $2,0^{\mathrm{b}}$ & \\
\hline & Peso aos 90 dias, $\mathrm{kg}$ & $10,0^{\mathrm{a}}$ & $10,8^{\mathrm{a}}$ & $7,3^{\mathrm{b}}$ & \\
\hline & Taxa de reprodução, \% & 64,3 & 64,3 & 60,0 & \\
\hline \multirow{6}{*}{$\begin{array}{c}\text { EM1 } \\
\text { (julho/agosto) } \\
\text { Parto: } \\
\text { dezembro/janeiro }\end{array}$} & Matriz exposta, $n$ & 11 & 13 & 20 & \multirow{6}{*}{$\begin{array}{l}\mathrm{P}<0,05 \\
\mathrm{P}<0,05\end{array}$} \\
\hline & Fertilidade ao parto, $\%$ & 54,5 & 76,9 & 70,0 & \\
\hline & Prolificidadem $\mathrm{n}$ & $1,2^{\mathrm{a}}$ & $1,3^{\mathrm{a}}$ & $1,1^{\mathrm{a}}$ & \\
\hline & Peso ao nascimento, $\mathrm{kg}$ & $3,8^{\mathrm{a}}$ & $2,6^{\mathrm{b}}$ & $2,3^{c}$ & \\
\hline & Peso aos 90 dias, $\mathrm{kg}$ & $12,5^{\mathrm{a}}$ & $11,9^{\mathrm{ab}}$ & $9,8^{\mathrm{b}}$ & \\
\hline & Taxa de reprodução, \% & 54,5 & 76,9 & 80,0 & \\
\hline \multirow{3}{*}{ Geral } & Peso ao nascimento, $\mathrm{kg}$ & $3,3^{\mathrm{a}}$ & $2,7^{b}$ & $2,1^{\mathrm{c}}$ & \multirow{3}{*}{---} \\
\hline & Peso aos 90 dias, $\mathrm{kg}$ & $11,9^{\mathrm{a}}$ & $11,2^{\mathrm{a}}$ & $8,0^{\mathrm{b}}$ & \\
\hline & Taxa de reprodução, \% & 53,8 & 63,7 & 68,3 & \\
\hline
\end{tabular}

a, b,c Letras minúsculas diferentes na mesma linha diferem pelo teste Tukey $(\mathrm{P}<0,05)$.

Não houve diferença significativa para as demais variáveis pelo teste do Qui-quadrado $(\mathrm{P}>0,05)$. 
Rev. Cient. Prod. Anim., v.12, n.1, p.60-63, 2010

O peso corporal médio das crias ao nascer variou de 2,08 $\mathrm{kg}$ nas crias Nativas a 3,3 $\mathrm{kg}$ paras as crias $1 / 2$ Boer/SRD. Em todas as estações de monta, as crias provenientes do cruzamento Boer x SRD ou Anglo x SRD foram mais pesadas $(\mathrm{P}<0,05)$ que as crias das raças nativas (Tabela 1). Esses resultados demonstram que reprodutores das raças Boer e Anglo-nubiana podem ser utilizados em cruzamentos industriais, promovendo maior peso das crias ao nascimento e ao desmame.

A duração do intervalo entre partos (IP) é um dos fatores para se avaliar a eficiência reprodutiva dos rebanhos. Na região semi-árida do Nordeste do Brasil, o IP tem-se mostrado superior a 300 dias para animais explorados extensivamente (Simplício et al., 1990). Em regime sem-intensivo com suplementação ou intensivo tem se recomendado a adoção de estações de monta para obtenção de três partos em dois anos, ou seja, com intervalo de parto de oito meses. Nesse trabalho, em dois anos de avaliação, foi observado um IP médio de 10 meses, ou seja, 1,2 partos/ano. Embora a fêmea caprina tenha o potencial reprodutivo de ter 1,5 partos/ano, em rebanhos comerciais na região semi-árida esse índice pode ser difícil ser alcançado.

Os resultados apresentados nesse trabalho mostram que o consumo exclusivo de pastagens pode não atender às exigências nutricionais de fêmeas caprinas antes e durante as estações de monta. Visando a otimização do desempenho reprodutivo, deve-se melhorar o aporte nutricional para que as cabras sejam fecundadas e possam ganhar peso, aumentando a taxa de ovulação e melhorando a taxa de fecundação.

\section{Conclusões}

O estabelecimento de estações de monta para obtenção de três partos em dois anos, em sistema de produção agroecológico no semi-árido, pode apresentar baixos índices zootécnicos se não houver um aporte nutricional para os animais. A utilização de cruzamentos industriais pode ser uma alternativa para melhorar a produção de carne de caprinos na região semi-árida do Nordeste do Brasil.

\section{Literatura Citada}

GUIMARÃES FLLHO, C. Eficiência reprodutiva de caprinos em zona semi-árida no Nordeste semiárido: limitações e possibilidades. Petrolina: Embrapa-CPATSA, 1983. 40p. (Embrapa-CPATSA. Documento, 20).

HOLANDA JÚNIOR, E.V., NOGUEIRA, D.M, DE ARAÚJO, G.G.L., MIRANDA, D.B., GUIMARÃES FILHO, C., REVOREDO, D.O. Desempenho do sistema de produção do "cabrito ecológico" no semi-árido: resultados do $1^{\mathrm{O}}$ ano. In.: 41 ${ }^{\text {a }}$ Reunião Anual da Sociedade Brasileira de Zootecnia (SBZ). CD Rom. Campo Grande, MS, 2004.

NOGUEIRA, D.M.; HOLANDA JÚNIOR, E.V. Desempenho reprodutivo de cabras 1/2 Boer e cabras sem raça definida em sistema de produção orgânica na região semi-árida do Nordeste do Brasil In.: $42^{\mathrm{a}}$ Sociedade Brasileira de Zootecnia (SBZ), CD Rom, Goiânia-GO, 2005.

NOGUEIRA, D.M.; MOREIRA, J.N.; VOLTOLINI, T.V.; PEREIRA, D.S.; GUIMARÃES FILHO, C. Variação do peso vivo durante a gestação e no pósparto de cabras exploradas em sistema de produção agroecológica na região semi-árida do Nordeste do Brasil. In.: 44 Reunião Anual da Sociedade Brasileira de Zootecnia (SBZ). CD Rom, Jaboticabal-SP. 2007.

SIMPLÍCIO, A.A., MACHADO, R., ALVES, J.U. Manejo reprodutivo de caprinos em regiões tropicais. In: Novas tecnologias de produção animal. SOCIEDADE BRASILEIRA DE ZOOTECNIA (SBZ) Anais... Piracicaba: FEALQ, p.109-132, 1990. 